\title{
Análisis de ideas de docentes de educación primaria sobre diversidad familiar
}

\author{
Analysis of Primary School Teachers' Opinions on family \\ Diversity
}

\section{Alvaro Capano Bosch ${ }^{1 a b^{*},}$ Natalie Massonnier ${ }^{1 a}$ \& Maria del L. González Tornaría $^{1 \mathrm{ac}}$}

\author{
${ }^{1}$ Universidad Católica del Uruguay, Montevideo, Uruguay.
}

assicólogo(a), Docente Universitario. ' Investigador Asociado al Departamento de Psicología del Desarrollo y Educación. 'Doctora en Ciencias de la Educación.

Recibido: $16-04-16$

Aprobado: 17-08-16

*Correspondencia

Email: alvarocapano@gmail.com
Citar como:

Capano, A., Massonnier N., \& González, M. (2016). Análisis de ideas de docentes de educación primaria sobre diversidad familiar. Propósitos y Representaciones, 4(2). 15-71. Doi: http://dx.doi.org/10.20511/pyr2016. v4n2.119

(c) Universidad San Ignacio de Loyola, Vicerrectorado de Investigación y Desarrollo, 2016. (e) BY.NC-ND Este artículo se distribuye bajo licencia CC BY-NC-ND 4.0 Internacional (http://creativecommons.org/licenses/by-nc-nd/4.0/). 


\section{Resumen}

Este artículo intenta indagar en las ideas de los docentes de educación primaria sobre los modelos familiares que se apartan del tradicional de familia nuclear. Se trabajó con 60 docentes de Montevideo y zona metropolitana respondiendo el Cuestionario de Ideas de los Docentes sobre Diversidad Familiar-CIDF-(Morgado, Jiménez-Lagares \& González, 2009). Los datos obtenidos refieren que los docentes no mantienen una visión homogénea de los distintos tipos de hogares. Las familias adoptivas, las de progenitores divorciados y las monoparentales bajo responsabilidad de madres tuvieron mejor aceptación entre los docentes que las homoparentales o las encabezadas en solitario por un padre. Los ítems relativos a familias homoparentales fueron respondidos por menos personas. Los docentes con formación de posgrado se mostraron más tolerantes y flexibles ante la diversidad familiar. Se discuten estos resultados a la luz de las necesarias adaptaciones en la educación primaria con respecto a la diversidad de familias que allí asisten.

Palabras clave: Diversidad familiar, ideas docentes, educación primaria.

\section{Summary}

This article aims to do an analysis based on the opinion of primary school teachers on family models that are different from the traditional nuclear family. We worked with 60 teachers from Montevideo and the metropolitan area. They answered the Questionnaire: Teachers' Opinion on Family Diversity (CIDF for its Spanish acronym) (Morgado, Jimenez-Lagares \& Gonzalez, 2009). The data indicate that teachers do not share a homogeneous point of view regarding the different household types. Adoptive families, divorced parents families, and single-parent families headed by single mothers had a better acceptance among teachers than homoparental families or those headed by single fathers. Items related to homoparental families were answered by fewer people. Teachers with postgraduate education were more tolerant and flexible towards family diversity. These results are discussed in light of the 
necessary adaptations in primary education with respect to the diversity of families existing there.

Keywords: Family Diversity, teachers' opinions, primary education. 


\section{Introducción}

La identificación de las creencias de los docentes sobre diferentes tópicos que hacen a la relación con sus alumnos y su evaluación se ha tenido en cuenta en las Ciencias de la Educación desde la famosa investigación de Rosenthal y Jacobson (1968), conocida como el Efecto Pigmaleón. Desde sus pioneros estudios, se ha verificado cuánto pesan las expectativas de logro o de fracaso de los docentes respecto de sus alumnos a la hora de evaluarlos.

Estas expectativas pueden estar relacionadas con las familias en que los niños crecen. En momentos en que se habla de inclusión educativa y de acogimiento de la diversidad, las distintas formas de familia de los alumnos no deberían quedar fuera de la consideración del concepto de inclusión.

Por lo tanto, teniendo en cuenta la creciente diversidad familiar, tanto en la configuración como en las dinámicas internas, resulta interesante el estudio de las ideas de los docentes sobre la variable que se maneja desde el inicio del vínculo con las familias, tal como es la configuración familiar.

La diversidad familiar aparece frecuentemente dentro de los temas que los docentes reportan como de mayor exigencia a la hora de relacionarse con las familias de los alumnos, influyendo en las prácticas cotidianas que tienen con ellas (González Tornaria, Wagner \& Saraiva Junges, 2015). Nos referimos al hecho de realizar una convocatoria para una reunión, citar a una entrevista, pedir colaboración para el seguimiento de un tratamiento o apoyar al niño en el estudio en la casa. Estos contactos exigen saber, de parte del docente, con quién vive el niño, cuál es el grado de compromiso de sus adultos referentes y con qué redes de apoyo cuentan.

Desde la perspectiva de los niños, la escuela constituye el primer escenario que les da la oportunidad de conocer otras realidades familiares diferentes a la suya, y este hecho se transforma en una variable que debe ser manejada por el docente.

Hoy nos encontramos frente a un panorama familiar de mayor complejidad, donde claramente se ha hecho un recorrido que nos lleva de 
"la familia modelo a los modelos de familias" (López, Díez, Morgado \& González, 2008, p. 112). Si tenemos en cuenta que en nuestra sociedad todos los niños, niñas y sus familias hacen su pasaje obligatorio por la escuela primaria, cabría preguntarnos: ¿Qué hace la institución educativa en la actualidad y que debería hacer frente a la diversidad? (López et al., 2008). Así como señala Pichardo (2011), hoy día en Uruguay las instituciones educativas parecen desconocer que no todos los referentes adultos son heterosexuales, que no todos los niños y niñas conviven en hogares de familias nucleares, subestimando de esta forma a las familias no convencionales y asumiendo la existencia de un modelo hegemónico que abarca a todo el alumnado (López et al., 2008). Una muestra de ello es la ausencia de la temática en los programas de formación docente.

No obstante lo manifestado, en los últimos años los materiales de lectura escolar han intentado explicitar la diversidad familiar, lo que garantiza su estudio en lo cotidiano. Si bien este hecho refleja la intención de tratamiento del tema, los docentes se encuentran desprovistos de recursos para su manejo en el aula, ya que no han recibido durante su formación insumos sobre temáticas relativas a familias y al vínculo a mantener con ellas (Administración Nacional de Educación Pública, 2010, 2012a, 2012b). Es así que uno de los puntos de mayor trascendencia con respecto a los cambios a realizar por la escuela primaria pasa por revisar las propias ideas de los docentes sobre la diversidad familiar, tal como refieren en su estudio Morgado, Jiménez-Lagares y González (2009). A partir de estos datos, se nos ocurre oportuno reflexionar sobre la importancia de trabajar no solo con los docentes de primaria sino también, por ejemplo, con los estudiantes de los centros de formación docente. En ese sentido, los cursos de formación docente que a nivel internacional incluyen la temática de las familias parten de la identificación de las creencias de los participantes inscriptos, no solo referidas a las configuraciones familiares, sino también a los posibles prejuicios que se puedan tener sobre la colaboración entre familias y escuelas (Deslandes, 2005). 
Los estudios pioneros acerca de las creencias docentes sobre configuración familiar pertenecen a Santrock y Tracy (1978). En esa oportunidad, ante un mismo video de un niño de 8 años, los docentes debían hacer una historia. Los que supusieron que era hijo de padres divorciados lo valoraron más bajo en felicidad, posibilidad de afrontamiento del estrés y ajuste emocional, que quienes partieron de la base de que ese niño crecía en una familia nuclear.

A partir de estos estudios se han desarrollado otros que, con diferentes metodologías y técnicas, han tratado de identificar estas ideas y cómo ellas pueden incidir en la relación que los docentes tienen con sus alumnos. Se ha visto que la voluntad de intervención por parte de los docentes, de haber una problemática en un niño, puede variar según se crea que es posible introducir un cambio o que esta dificultad se debe a la especial configuración familiar. Es así que la posibilidad de relacionarse con las familias de los alumnos en forma saludable, desde la posibilidad de informarlas hasta crear instancias de participación, ha sido destacada como una de las competencias imprescindibles para los docentes en la actualidad (Perrenoud, 2004).

Entre las últimas investigaciones efectuadas al respecto, Triana y Rodrigo (2010) reportan un estudio realizado por su equipo en 2007, en el que se exploraron las expectativas de los docentes sobre adaptación emocional, social y académica de los alumnos, según la configuración familiar a la que pertenecieran. Trabajaron contrastando las expectativas respecto de alumnos de familias nucleares, monoparentales de jefatura femenina producto de una separación, reconstituidas, adoptivas y homoparentales. Encontraron que quienes tenían las mejores predicciones en todas las áreas eran los hijos de familias nucleares, seguidos por los que pertenecían a familias adoptivas. Los niños que vivían en hogares a cargo de mujeres y en hogares reconstituidos tuvieron, a su vez, expectativas más favorables que aquellos que pertenecían a hogares homoparentales. Las autoras adjudican las expectativas desfavorables respecto a este último tipo de familias a que ellas son las menos comunes tanto a nivel del contexto escolar como social. Este estudio deja clara la necesidad de identificar estas expectativas, ya que ellas 
pueden afectar la interacción y la evaluación que los docentes hacen de sus alumnos.

Estudios referidos a las relaciones entre escuelas y familias (Comellas, 2009; Núñez, 2003; Oliva \& Palacios, 2005) han dejado clara la importancia de las percepciones que los docentes tienen sobre las configuraciones familiares de sus alumnos, no solo en la evaluación de los niños, sino en la forma como encaran la relación con sus familias y en la valoración que hacen del currículum educativo familiar, entendido como el proyecto educativo que los padres concretan basados en sus percepciones y creencias sobre la crianza de los hijos (Rodrigo \& Acuña, 2005).

Petricone (2009), por su parte, ha estudiado la influencia de la historia familiar de los docentes en sus creencias y estilos profesionales. Trabajó con 170 docentes de Madrid y encontró que los estilos educativos identificados en sus familias de origen se agruparon en funcionales (63.7\%) y disfuncionales (36.3\%). Estos estilos condicionan los estilos educativos de los docentes, pudiendo diferenciarse tres tipos: flexibles y democráticos, tendientes a la rigidez y autocracia, y rígidos y autocráticos. En esta investigación, 69.5\% de los docentes reportaron que el estilo educativo de las familias de origen incidía mucho en su propio estilo docente, mientras que el 30\% opinó que solo algo y el $0.5 \%$ restante que nada.

Entre las investigaciones efectuadas respecto a la temática de ideas docentes sobre diversidad familiar, es muy interesante la de Morgado, Jiménez-Lagares y González (2009). Las autoras reportan que, en gran parte de los estudios, los docentes asociaban mejor competencia académica, social y ajuste emocional en niños y niñas que provenían de una pareja casada. Asimismo, de la misma investigación surge que una parte del profesorado desconoce algunos modelos familiares no convencionales o mantiene importantes prejuicios frente a ellos.

Sin embargo, investigaciones recientes (Oliva \& Arranz, 2011) que se llevaron a cabo con familias de diferentes configuraciones: nucleares, monoparentales, homoparentales, reconstituidas, con embarazos múltiples y 
adoptivas, contradicen las creencias antes reportadas. Se hizo uso de técnicas tales como entrevista, observación y cuestionarios. En sus resultados, dejan en claro que la diversidad en la configuración familiar no resulta significativa en el desarrollo del niño, salvo que se aglutinen otras variables. Estas variables son las relacionadas con clima familiar, tales como manejo del conflicto marital, afrontamiento del estrés y estilos educativos, las que se ha comprobado que tienen el mayor peso en el ajuste infantil.

Por su parte, Golombok (2006) sostiene que las características familiares fundamentales para un desarrollo psicológico saludable son: la seguridad afectiva, el bajo nivel de conflicto marital, la existencia de un estilo educativo democrático, la calidad de la interacción y de la estimulación entre el niño/a y sus padres y la frustración óptima Esta autora refiere que, en un contexto familiar donde se cumple con las características mencionadas anteriormente, se dan las condiciones para una crianza con total confiabilidad, más allá de la configuración familiar. Asimismo, Arranz, Oliva, Olabarrieta y Antolín (2010) concluyen que no es la configuración, sino las variables sociodemográficas y las variables interactivas, las que influyen en la calidad del contexto familiar.

Cabría preguntarse entonces qué garantiza un buen desarrollo y aprendizaje a nivel familiar. Rodrigo (2001) menciona la necesidad de un importante nivel de afectividad en el hogar, relaciones de apoyo, seguras y de confianza, una buena comunicación, un estilo de socialización variado y adecuado a la situación y comportamientos de los hijos, un ambiente que estimule el aprendizaje, la implicación y participación de los padres en la educación en el hogar y en el centro educativo; y, por último, la estimulación para el desarrollo de la educación para el ocio y la promoción de estilos de vida saludables. Igualmente, en la lista de variables contextuales optimizadoras del desarrollo psicológico, Arranz y Oliva (2010) destacan la estabilidad laboral, la posibilidad de acceso a recursos para la estimulación del desarrollo, la existencia de redes de apoyo familiar y social, espacios físicos adecuados en el hogar y en el barrio, así como relaciones positivas con el centro educativo y ausencia de situaciones estresantes. Cuando enumeran 
las variables interactivas, ponen acento en la presencia, la estimulación y el interés parental en los asuntos concernientes a los niños, la práctica de estilos educativos democráticos y un bajo nivel de conflicto. En ningún caso se hace mención a un tipo de configuración familiar en particular.

En Uruguay, indagar sobre la postura de los docentes y futuros docentes frente a las diferentes configuraciones familiares se vuelve fundamental, teniendo en cuenta los grandes cambios de las familias en las últimas décadas.

Los divorcios aumentaron, pasando de una tasa de $18.7 \%$ para el año 1985 a una tasa de 33.7\% en el año 2002 (Cabella, 2007). Los hogares monoparentales tuvieron un incremento: en 1996 alcanzaban un $9.6 \%$ y en el 2011 constituían un $11.1 \%$ de los hogares relevados. Los hogares compuestos por parejas con hijos decrecieron, pasando de un $34.1 \%$ en 1996 a un $31.4 \%$ en 2011, mientras que los hogares extendidos bajaron en el mismo periodo de 20.1\% a 14.9\% (Instituto Nacional de Estadística, 1996, 2011). Según datos del último censo, 2778 personas conviven en pareja con personas del mismo sexo (INE, 2011). En las primeras décadas del siglo XXI, 80\% de los jóvenes prefieren la unión libre al matrimonio. En 2010, 70\% de los nacimientos se dan fuera del matrimonio.

En el censo de 2011, el 14.1\% de la población son personas de 65 años y más, siendo esta cifra la más alta de América del Sur (Cabella, Fernández Soto \& Prieto, 2015). Las mujeres entre 25 y 62 años han aumentado su tasa de actividad laboral, que alcanza un $75 \%$, con variaciones según tramo de edad y nivel educativo (Nathan \& Zerpa, 2015). Según la Encuesta de Situaciones Familiares de 2008, realizada con 1399 hijos menores de 22 años, $16.7 \%$ viven con su madre, estando su padre vivo; $33 \%$ de ellos no ve nunca a su padre, mientras que $40 \%$ nunca recibieron transferencias económicas (Bucheli \& Vigorito, 2015). A nivel legislativo, se han dado varios cambios que han implicado el reconocimiento de la diversidad de situaciones parentales: la Ley 18246 de Unión Concubinaria, en 2008, y la Ley 19075 de Matrimonio Igualitario, que modifica disposiciones relativas a la adopción, en 2013. 
Teniendo en cuenta los datos antes señalados, se vuelve necesario implementar cambios tanto a nivel de la formación de los docentes como a nivel del vínculo con las familias en las escuelas.

Es importante consignar que en Uruguay no se han realizado publicaciones previas en la línea de este trabajo. Por tal motivo, la elaboración del presente estudio es, en sí misma, un aporte novedoso que podría contribuir de forma significativa al sistema educativo, enriqueciendo la reflexión y la discusión, y aportando elementos para el cambio en los programas educativos y la praxis docente.

\section{Objetivos.}

- Analizar las ideas de los docentes de educación primaria acerca de las familias no convencionales.

- Indagar si existen diferencias en las ideas de los docentes acerca de la diversidad familiar en función de la edad, la antigüedad docente, el tipo de centro en el que imparten docencia, su configuración familiar y el nivel de formación académica de los docentes.

\section{Método}

\section{Participantes.}

Se trata de un total de $(n=60)$ docentes que trabajan en instituciones educativas privadas y públicas. La elección de los centros escolares para este estudio se realizó en base a contactos preexistentes con personas dentro de las instituciones, producto de la realización de otras investigaciones ejecutadas por el mismo equipo. Con respecto a los docentes, la muestra fue incidental. De estos docentes, el $98.3 \%$ son del sexo femenino y el $1.7 \%$ del sexo masculino. La edad media de los docentes del estudio es de 39 años y 6 meses, alcanzando una edad máxima de 65 y una mínima de 23 años. En cuanto al ejercicio de la docencia, la media para los 58 docentes que respondieron es de 16 años y 8 meses. 
En relación con la constitución familiar, cabe consignar que un $6.7 \%$ de las mujeres viven en unión libre, $36.7 \%$ han conformado una familia nuclear, $25 \%$ viven con sus familias de origen, $3.3 \%$ conviven en familias extensas, $13.3 \%$ en hogares monoparentales, $10 \%$ en hogares unipersonales y $3.3 \%$ no contestan. En cuanto al docente hombre encuestado, convive en una familia nuclear.

Con respecto a la filiación institucional de los docentes, $41.7 \%$ de ellos trabajan exclusivamente en educación privada católica, mientras que 33.3\% comparte su inserción entre la educación privada católica y la educación pública, $8.3 \%$ trabaja exclusivamente en educación privada laica y $16.7 \%$ comparte su labor docente entre la educación privada laica y la educación pública.

\section{Tabla 1.}

Características de la muestra de docentes.

\begin{tabular}{|c|c|c|c|c|}
\hline \multicolumn{5}{|c|}{ Muestra de docentes estudiados $(n=60)$} \\
\hline Edad & $\bar{X}=39.67$ años & $\mathrm{DT}=12.25$ & Mínimo: 23 & Máximo: 65 \\
\hline Años de docencia & $\bar{X}=16.78$ años & $\mathrm{DT}=12.25$ & Mínimo: 2 & Máximo: 44 \\
\hline \multirow{2}{*}{ Sexo } & \multicolumn{4}{|c|}{ Mujer $98.3 \%(\mathrm{n}=59)$} \\
\hline & \multicolumn{4}{|c|}{ Hombre $1.7 \%(\mathrm{n}=1)$} \\
\hline \multirow{4}{*}{ Colegio } & \multicolumn{4}{|c|}{ Privado Católico $41.7 \%(n=25)$} \\
\hline & \multicolumn{4}{|c|}{ Privado Laico $8.3 \%(\mathrm{n}=5)$} \\
\hline & \multicolumn{4}{|c|}{ Escuela Pública/Privado Católico 33.3\% ( $\mathrm{n}=20)$} \\
\hline & \multicolumn{4}{|c|}{ Escuela Pública/Privado Laico 16.7\% $(\mathrm{n}=10)$} \\
\hline Familiar & \multicolumn{4}{|c|}{ Familia Nuclear 38.3\% $(\mathrm{n}=23)$} \\
\hline \multirow{6}{*}{ Configuración } & \multicolumn{4}{|c|}{ Familia Monoparental 13.3\% $(\mathrm{n}=8)$} \\
\hline & \multicolumn{4}{|c|}{ Familia Extensa 3.3\% $(n=2)$} \\
\hline & \multicolumn{4}{|c|}{ Familia de Origen $25 \%(n=15)$} \\
\hline & \multicolumn{4}{|c|}{ Unipersonal 10\% $(\mathrm{n}=6)$} \\
\hline & \multicolumn{4}{|c|}{ Pareja s/h Unión libre 6.7\% $(n=4)$} \\
\hline & \multicolumn{4}{|c|}{ No contestan 3.3\% (n : 2) } \\
\hline
\end{tabular}


Como lo muestra la Tabla 1, los participantes son mayoritariamente mujeres, coincidiendo con lo señalado en el censo realizado en los años 2014 y 2015, donde 9 de cada 10 estudiantes de magisterio resultaron ser de sexo femenino. En lo que respecta al ejercicio profesional, en el año 2007 la cantidad de maestras en ejercicio alcanzaba el 93.1\% del total de docentes de primaria (Administración Nacional de Educación Pública, 2015). Las medias nos indican que, en cuanto a edad, los participantes son maduros, y en cuanto a ejercicio profesional, cuentan con experiencia, habiendo superado las primeras etapas de vida laboral.

\section{Instrumentos y procedimiento.}

En este estudio se ha replicado el diseño de investigación de carácter exploratorio presentado por Morgado et al. (2009) y se ha aplicado el instrumento Cuestionario de Ideas acerca de la Diversidad Familiar (CIDF) de Morgado et al., (2009) con su autorización. Se trata de una escala autoadministrada, compuesta por 15 ítems, que se contesta en una escala de Likert, que va desde " 1 " (totalmente en desacuerdo) hasta "4" (totalmente de acuerdo), escala par que fuerza las respuestas de los docentes. Los análisis de consistencia interna de la escala en el estudio español informaron que el ítem 14 mantenía una correlación inversa con el total, por lo que se procedió a su eliminación en esos análisis. El índice de fiabilidad obtenido para la escala, una vez eliminado dicho ítem, fue medio-alto (Alfa de Cronbach basada en valores tipificados $=0.75$ ).

El análisis de consistencia interna de la escala para nuestro estudio fue medio alto (Alfa de Cronbach basada en valores tipificados $=0.71$. No se procedió a la eliminación del ítem 14 como en el estudio español, ya que la modificación en el índice de Alfa de Cronbach en valores tipificados no se veía considerablemente afectado, siendo $=0.73$ sin el ítem 14). Además de haber tenido en cuenta las cualidades reseñadas, se decidió trabajar con este instrumento porque aborda varias formas de diversidad familiar y es de fácil aplicación, se completa en pocos minutos y puede ser administrado en 
forma grupal. Asimismo, se eligió este instrumento debido a la pretensión de replicar la investigación mencionada.

La recolección de los datos se llevó a cabo entre el segundo semestre del año 2013 y el primer semestre del año 2014, en Montevideo y zona metropolitana. En todos los casos, los sujetos completaron el consentimiento informado. Una vez completado dicho documento y planteadas las preguntas que surgieran, los sujetos llenaron el cuestionario que acompañaba el CIDF y que solicitaba sus datos sociodemográficos.

\section{Resultados}

Para cumplir con el primer objetivo y analizar las ideas de docentes de primaria acerca de familias no convencionales, se agruparon las respuestas que marcaron acuerdo, tanto total como simple. En cuanto al ítem 6, va en línea con el estudio español (90.5\%), alcanzando un guarismo aún mayor: $96.7 \%$ de los docentes están de acuerdo o totalmente de acuerdo en que lo importante no es la configuración familiar, sino lo feliz que pueda sentirse el niño en su hogar. Al igual que en el estudio de Morgado et al. (2009) pareciera, en un primer análisis, que los docentes valoran la relación familiar por sobre la configuración. En esa misma línea encontramos las investigaciones realizadas por Arranz y Oliva (2010), Arranz et al. (2010), Golombok (2006), Oliva y Arranz (2011).

Como vemos en la Tabla 2, y en relación con las familias adoptivas, el 98.3\% de los docentes entiende que los hijos(as) adoptados pueden ser igual de queridos que los hijos(as) biológicos; mientras que el 15.3\% piensa que los hijos adoptados tendrán problemas irrecuperables. Hay una alta similitud con el estudio español en ambos ítems (93.4\% y 14.6\%, respectivamente). En línea con el hecho de ser los hijos/as adoptados igual de queridos que los biológicos, Bernedo, Fuentes, Fernández-Molina y Bersabé (2007) refieren una alta percepción en familias adoptivas, tanto en afecto como en comunicación y en un estilo parental inductivo, y menos críticas e indulgentes. 
En ese sentido, el estudio de Triana y Rodrigo (2010) indica que las familias adoptivas contaban con las mejores predicciones en cuanto al desarrollo de los niños de parte de los docentes, inmediatamente luego de las familias nucleares.

Con respecto a los ítems relacionados con el divorcio, el 61\% de los docentes están de acuerdo en que los hijos(as) de padres separados se encuentran marcados por esa separación, mientras que en el estudio español alcanza un $77.4 \%$, evidenciándose una diferencia en este ítem. Este punto resulta importante si tenemos en cuenta el aumento significativo de divorcios en nuestro país (Cabella, 2007). Asimismo, vemos que el $79.3 \%$ de los docentes piensan que, luego de pasado un tiempo de la separación, los niños y niñas alcanzan la normalidad; y en el estudio español, ese porcentaje alcanzó el 63.7\%, habiendo también diferencia. En tanto, el 96.6\% piensa que, después de la separación, los niños y niñas pueden mantener un contacto estrecho y cálido con los padres, mientras que, en el estudio español, esta cifra fue del $85.4 \%$. Debemos tener presente que, en Uruguay, el 33\% de los menores de 22 años no ve a su padre luego de la separación y un $40 \%$ no recibe transferencia económica (Bucheli \& Vigorito, 2015).

En cuanto a las familias monoparentales, el 58.6\% de los docentes piensa que la ausencia de la madre genera problemas en el desarrollo de los niños/as, mientras que, en el estudio español, el porcentaje asciende a $61 \%$ de la muestra. En este sentido destacamos que el $87 \%$ de los hogares monoparentales en nuestro país cuentan con jefatura femenina (INE, 2011). Se debe tener en cuenta que, en el estudio de Triana y Rodrigo (2010), la monoparentalidad con jefatura femenina era considerada de riesgo para la adaptación emocional, social y académica de los niños y niñas. Por su parte, el $10 \%$ de los docentes cree que la ausencia del padre tiene efectos negativos para el desarrollo de los hijos/as, siendo el $22 \%$ en el estudio español. Asimismo, la necesaria presencia de la figura paterna para que un niño sea adecuadamente masculino, según la muestra de docentes uruguayos, alcanza un guarismo de $25.5 \%$, siendo este superior al estudio español, con un $10.7 \%$. 
En relación con los hogares homoparentales, y de acuerdo con la muestra consultada, cabe consignar que el $16 \%$ de los docentes creen que los niños y niñas no deberían ser adoptados por homosexuales ya que habría mayor probabilidad de que tengan problemas en su desarrollo. Mientras que el $58.8 \%$ de las docentes consultadas creen que los hijos e hijas de gays y lesbianas no se diferencian en nada de los hijos e hijas de heterosexuales. En tanto, un $42.6 \%$ de las docentes concuerdan en que los niños y niñas que viven con gays y lesbianas sufren el rechazo social. En lo que respecta a los hogares homoparentales, es necesario recordar que en Uruguay, a partir de la creación de la Ley 19075 de Matrimonio Igualitario (2013), se modifican las disposiciones en torno a las adopciones, otorgando idénticos derechos en materia de adopción y reproducción asistida a las parejas de igual y distinto sexo.

El resultado del estudio hispano en relación con el primer ítem de homoparentalidad se encuentra considerablemente alejado del obtenido en el presente estudio, alcanzando un $42.4 \%$. Los otros ítems obtienen resultados más cercanos con $53 \%$ y $37.6 \%$, respectivamente.

En cuanto a los ítems relacionados con los hogares biparentales, un $15.3 \%$ de los docentes cree que los hogares con padres que trabajan y una madre que se queda en casa es el mejor entorno de desarrollo para los hijos(as). Asimismo, un 15.3\% concuerdan en que, cuando las madres trabajan fuera del hogar, los hijos/as tienen más probabilidades de padecer problemas escolares. En el estudio español, las cifras coinciden para ambos ítems, contando con el acuerdo de uno de cada cuatro docentes. Por lo tanto, respecto a las consecuencias en el desarrollo de los niños, nuestros docentes ven menos riesgos que los españoles. Esto puede explicarse por la gran inserción laboral de la mujer, que alcanza un 75\% entre los 25 y los 62 años, pero que aumenta a un $90 \%$ en el caso de la población que ha alcanzado educación terciaria (Nathan \& Zerpa, 2015). Este es el caso de nuestra muestra que, además, tiene una composición femenina que llega al 98.3\%. 
Al igual que en el estudio de Morgado et al. (2009), queda de manifiesto que los docentes no tienen una visión homogénea de los diferentes tipos de hogares.

\section{Tabla 2.}

Resultados del porcentaje de acuerdo, la puntuación media, la desviación típica (DT) y el porcentaje de respuestas a cada item del Cuestionario de Ideas acerca de la Diversidad Familiar (CIDF).

\begin{tabular}{|c|c|c|c|c|c|}
\hline & Ítems & $\begin{array}{l}\% \text { Acuerdo } \\
\text { o total } \\
\text { acuerdo }\end{array}$ & Media & DT & $\%$ Respuestas \\
\hline $\begin{array}{l}\text { Dinámica } \\
\text { familiar }\end{array}$ & $\begin{array}{l}\text { Ítem 6: Lo importante para el } \\
\text { niño/a no es con quién vive sino } \\
\text { lo feliz que se siente en ese hogar. }\end{array}$ & 96.7 & 3.62 & 0.61 & 100 \\
\hline $\begin{array}{l}\text { Familias } \\
\text { ambos } \\
\text { progenitores } \\
\text { trabajan }\end{array}$ & $\begin{array}{l}\text { Ítem 5: Un padre que trabaja y } \\
\text { madre en casa es el mejor entorno } \\
\text { para el desarrollo de hijos/as (i) } \\
\text { Ítem 13: Cuando las madres } \\
\text { trabajan fuera del hogar, hijos/ } \\
\text { as tienen más probabilidad de } \\
\text { padecer problemas escolares (i) }\end{array}$ & $\begin{array}{l}15.3 \\
15.3\end{array}$ & $\begin{array}{l}3.19 \\
3.17\end{array}$ & $\begin{array}{l}0.73 \\
0.77\end{array}$ & $\begin{array}{l}98.3 \\
98.3\end{array}$ \\
\hline $\begin{array}{l}\text { Familias } \\
\text { adoptivas }\end{array}$ & $\begin{array}{l}\text { Ítem 2: Hijos e hijas } \\
\text { adoptados presentan problemas } \\
\text { irrecuperables (i) } \\
\text { Ítem 9: Hijos e hijas adoptados } \\
\text { pueden ser igual de queridos que } \\
\text { biológicos }\end{array}$ & $\begin{array}{l}15.3 \\
98.3\end{array}$ & $\begin{array}{c}3.08 \\
3.8\end{array}$ & $\begin{array}{l}0.62 \\
0.51\end{array}$ & $\begin{array}{c}98.3 \\
100\end{array}$ \\
\hline $\begin{array}{l}\text { Separación } \\
\text { o divorcio }\end{array}$ & $\begin{array}{l}\text { Ítem 1: La mayoría de los hijos } \\
\text { o hijas de padres separados están } \\
\text { marcados por la separación (i) } \\
\text { Ítem 10: Tras un tiempo de la } \\
\text { separación, hijos e hijas recuperan } \\
\text { la normalidad } \\
\text { Ítem 12: Tras la separación, } \\
\text { padres e hijos pueden mantener } \\
\text { relación estrecha y cálida }\end{array}$ & $\begin{array}{c}61 \\
79.3 \\
96.6\end{array}$ & $\begin{array}{l}2.37 \\
2.93 \\
3.59\end{array}$ & $\begin{array}{l}0.72 \\
0.65 \\
0.62\end{array}$ & $\begin{array}{l}98.3 \\
96.6 \\
98.3\end{array}$ \\
\hline
\end{tabular}


Ítem 4: La ausencia del padre tiene efectos negativos sobre el desarrollo de hijos/as (i) Ítem 7: La figura paterna es

$\begin{array}{llcccc} & \text { necesaria para que un niño sea } & 10 & 3.32 & 0.75 & 100 \\ \text { Familias } & \text { adecuadamente masculino (i) } & 25.5 & 3.04 & 0.79 & 91.6 \\ \text { monopa- } & \text { Ítem 11: La ausencia de la } & 58.6 & 2.52 & 0.71 & 96.6 \\ \text { rentales } & \text { madre afecta irremisiblemente el } & 24.5 & 2.16 & 0.62 & 95\end{array}$
desarrollo de los hijos/as (i)

Item 14: Los niños y niñas que crecen solo con sus madres maduran antes

\begin{tabular}{|c|c|c|c|c|c|}
\hline $\begin{array}{l}\text { Familias } \\
\text { homopa- } \\
\text { rentales }\end{array}$ & $\begin{array}{l}\text { Ítem3: Homosexuales no } \\
\text { deberían poder adoptar, sus hijos } \\
\text { tienen más probabilidad de tener } \\
\text { problemas de desarrollo (i) } \\
\text { Ítem 8: Los hijos e hijas de gays } \\
\text { y lesbianas no se diferencian en } \\
\text { nada de los hijos e hijas de padres } \\
\text { y madres heterosexuales } \\
\text { Ítem 15: Niños y niñas que } \\
\text { viven con gays y lesbianas } \\
\text { necesariamente sufren rechazo } \\
\text { social (i) }\end{array}$ & $\begin{array}{c}16 \\
58.8 \\
42.6\end{array}$ & $\begin{array}{l}3.09 \\
2.65 \\
2.63\end{array}$ & $\begin{array}{l}0.84 \\
0.72 \\
0.56\end{array}$ & $\begin{array}{c}93.3 \\
85 \\
90\end{array}$ \\
\hline
\end{tabular}

\section{Presencia/ausencia de respuestas.}

En cuanto a la ausencia de respuestas, y siguiendo a Morgado et al. (2009), vemos que coinciden los ítems con bajo porcentaje de respuesta. Estos son los relacionados con familias homoparentales, tanto el ítem 8 como el 15. Los porcentajes alcanzados para ambos ítems son un $85 \%$ y un $90 \%$, respectivamente. Los resultados obtenidos en el estudio no son tan bajos como los que surgen del estudio español; el porcentaje de respuestas en ese estudio es de un $78.7 \%$ para ambos ítems. Este bajo porcentaje de respuestas puede deberse a lo ya reportado en otros estudios (Triana \& Rodrigo, 2010), relativo a que se trata de una configuración aún poco frecuente y conocida en el contexto escolar, lo que puede provocar la abstención de quienes responden. 


\section{Tabla 3.}

Contraste entre la frecuencia de respuestas en los items relativos a familias homoparentales versus el resto de items.

\begin{tabular}{lccc}
\hline & \multicolumn{2}{c}{ Presencia de la respuesta } & Total \\
\hline $\begin{array}{l}\text { Ítems familias homo- } \\
\text { parentales }\end{array}$ & $161(89.5 \%)$ & $19(10.5 \%)$ & 180 \\
\hline Resto de ítems & $703(97.6 \%)$ & $17(2.4 \%)$ & 720 \\
\hline Total & $864(96 \%)$ & $36(4 \%)$ & 900 \\
\hline
\end{tabular}

\section{Diferencias entre los docentes.}

A efectos de alcanzar el segundo objetivo, en lo que respecta a la edad de los docentes y su antigüedad, no aparecen relaciones significativas. La matriz de correlaciones nos informa que las ideas acerca de la diversidad familiar mostraron una relación negativa, que no es significativa con respecto a la edad $\left(r^{2}=-0.167 ; p=0.269\right)$ y a la antigüedad docente $\left(r^{2}=-0.128 ; p=0.408\right)$.

Con respecto al tipo de centro donde trabajan los docentes, no encontramos diferencias significativas para la variable ideas de los docentes sobre la diversidad familiar, según se desempeñen en: Público - Privado Católico (=42.19), Público - Privado Laico (=44.57), Privado Católico $(=43.85)$ y Privado Laico $(=41.33)$, arrojando el ANOVA $(F=0.830$ y $p=0.485)$.

Al comparar las ideas del profesorado sobre diversidad familiar entre quienes han alcanzado formación de posgrado $(=44.44)$ y quienes no $(=42.83)$, las diferencias que encontramos no son estadísticamente significativas, siendo los resultados para la igualdad de varianzas en la prueba de Levene $(F=1.69$ y $p=0.20)$ y para la prueba $\mathrm{T}$ ( $t=0.98$ y $p=0.33)$. Al realizar el análisis por ítem, únicamente hallamos diferencias significativas, 
entre quienes tienen formación de posgrado y quienes no, en el Ítem 6 ("lo importante para un niño/a no es con quién vive, sino lo feliz y seguro que se siente en ese hogar"), siendo los resultados de la prueba de Levene ( $p=.001)$ y para la prueba $\mathrm{t}(p=.013)$.

Al comparar mediante la prueba ANOVA si existen diferencias en la percepción de las ideas del profesorado según la configuración familiar, no encontramos diferencias significativas $(p=0.409)$.

Estos resultados marcan diferencias con los obtenidos en el estudio español, que arrojó relación negativa altamente significativa entre las ideas respecto a la diversidad familiar y la edad y experiencia docente de los participantes.

En ese sentido, hay que tener en cuenta que los docentes españoles en sus medias son más grandes y experimentados que los uruguayos, lo que puede explicar en alguna medida su reticencia hacia nuevas formas de familia, surgidas en forma acelerada en los últimos años. En cuanto a ejercicio laboral, cuentan con una media de 21 años, lo que indicaría que están más adentrados que los uruguayos en la cuarta etapa de vida profesional ubicada por Day (2005), entre los 16 y 23 años, marcada por tensiones en cuanto a la motivación y al compromiso profesional debido, principalmente, al necesario equilibrio que deben realizar entre vida personal y laboral.

\section{Discusión}

Resulta interesante, y se encuentra en consonancia con múltiples investigaciones hechas con otras poblaciones (Antolín, Oliva \& Arranz, 2009; Arranz, Oliva, Olabarrieta \& Antolín, 2010; Golombok, 2006; González, Chacón, Gómez, Sánchez y Morcillo, 2002; Oliva \& Arranz, 2011), que los docentes en este estudio piensen que lo importante para un niño/a no es con quién vive sino lo feliz que se siente en ese hogar, destacando la calidad de los contextos familiares y no la configuración familiar. 
Si se analiza la formación docente, es significativa la diferencia en relación con el ítem 6 , resultando que los docentes con mayor nivel académico se presentan más flexibles y tolerantes a la diversidad familiar. Este hecho abre una puerta interesante a las posibilidades que brindan tanto la formación inicial como la actualización permanente.

Con respecto a las respuestas encontradas en torno a las familias monoparentales, a la ausencia de la madre, del padre y a la necesaria presencia de este para que los niños sean adecuadamente masculinos, entendemos que esto genera una tensión en relación con el importante crecimiento que han tenido estos arreglos familiares en Uruguay, producto básicamente del aumento del divorcio (Cabella 2007). Asimismo, tengamos en cuenta que las respuestas de docentes con respecto a la ausencia de la madre, en un alto porcentaje, como factor que perturba el desarrollo de niños y niñas, y a la ausencia del padre, en un porcentaje menor, genera una incongruencia con la importancia que se le da en el ítem 6 al hecho de sentirse feliz con quien se vive y no a la configuración familiar. Estos datos se encuentran en consonancia con los expuestos en su estudio por Moliner y Francisco (2016).

Además, en cuanto a la ausencia del padre y sus consecuencias en la masculinidad del hijo, esto puede relacionarse con la preocupación de los docentes ante el hecho de que, en nuestro país, al menos un tercio de los niños dejan de ver a su padre y no reciben transferencias económicas luego del divorcio. Nuestra muestra es ampliamente femenina, y se ha comprobado en estudios sobre el tema en Uruguay que las mujeres, quienes en general quedan a cargo de los hijos, son más severas que los hombres a la hora de reportar datos y evaluar las consecuencias del divorcio en los niños (Bucheli \& Vigorito, 2015).

Si bien el acceso a la monoparentalidad puede expresar objetivamente situaciones similares, desde el punto de vista subjetivo acarrea experiencias y vivencias singulares (Jociles, Rivas, Moncó, Villamil \& Díaz, 2008). Señalamos aquí una de las limitaciones del instrumento, ya que los ítems 
referidos a monoparentalidad pueden reflejar múltiples situaciones, difíciles de tener en cuenta en su complejidad a la hora de contestar.

En relación con lo indagado por el cuestionario con familias divorciadas, cabe señalar que seis de diez docentes manifiestan que los hijos de estas familias se encuentran marcados por la separación. En el estudio de Oliva y Arranz (2011) surge que estas familias relatan que el proceso de separación fue conflictivo, y expresan su preocupación por el impacto del mismo en los niños. Sin embargo, los autores no hallaron una asociación significativa con el nivel de conflicto ni problemas con el ajuste infantil. Con respecto a las familias homoparentales y su inserción en las instituciones educativas, cuatro de diez docentes de nuestro estudio están de acuerdo con la posibilidad de rechazo social hacia estos niños y niñas. Justamente, Arranz, Oliva, Martin y Parra (2010) indican que estas familias anuncian en el centro educativo su condición de familia homoparental con el objetivo de buscar apoyo en la institución y evitar que sus hijos reciban el rechazo o acoso de sus compañeros. El hecho de omitir la condición de familia homoparental puede afectar la construcción de la identidad de los niños y provocar el acoso moral o psicológico (Mello, Grossi \& Uziel, 2009).

Por lo tanto, es esperable también que en nuestro país se produzcan cambios en este sentido, aunque todavía es temprano para tener datos al respecto debido a lo reciente de la Ley de Matrimonio Igualitario y de la posibilidad de adopción por parejas del mismo sexo.

A propósito de la relación familias homoparentales y escuelas, es interesante un estudio realizado por Ceballos-Fernández (2009) que refiere una discontinuidad entre las experiencias educativas en el marco de estas familias y las vividas en el centro educativo. Se vuelve entonces imperativa la interacción entre el sistema de educación formal y la educación familiar, el trabajo con metas comunes, reconociendo el principio de responsabilidad educativa compartida o corresponsabilidad (Marcondes y Sigolo, 2012; Torío, 2004), como forma de evitar la desconexión y el desequilibrio entre ambos contextos (Ceballos-Fernández, 2009). 
En una investigación sobre hijos e hijas de familias homoparentales que refiere a diversos aspectos de la vida familiar, se concluye que estos llevan una vida normalizada en cuanto a actividades cotidianas y cuentan con relaciones familiares caracterizadas por la comunicación, el afecto, la escasez de conflictos y los contactos frecuentes y cálidos con la familia extensa (González \& López, 2009). En este sentido, debemos considerar que la orientación sexual de los padres y madres no es un indicador para evaluar la función educadora de estos (Ceballos-Fernández, 2012).

En lo que concierne a la adopción de niños por parte de parejas homosexuales (ítem 3), existe menor rechazo en la muestra de docentes uruguayos (16\%) que en la de docentes españoles (42.3\%). En este caso, si bien es reciente la legislación, consideramos que puede estar influyendo tanto en un cambio de ideas respecto de esta posibilidad como en la deseabilidad social en torno a la respuesta. Las contestaciones relativas a configuraciones homoparentales reflejan en nuestro estudio, así como en el de Morgado et al. (2009) y en el de Triana y Rodrigo (2010), la actual reticencia respecto de estas familias, que se puede traducir en rechazo, bajo nivel de expectativas o en abstención cuando se les pregunta sobre ellas.

La visión más indulgente respecto del trabajo femenino por parte de los docentes uruguayos puede relacionarse con una visión un tanto más alejada de la familia nuclear tradicional en comparación con el caso de los docentes españoles. Esto se comprueba no solo en los ítems relativos al trabajo femenino, sino también en los que refieren a las familias divorciadas, a la ausencia del padre y sus consecuencias en el desarrollo de los hijos, y a la posibilidad de adopción por parte de parejas homosexuales. En esa misma línea, en el ítem 6 , que privilegia la dinámica familiar sobre la configuración, los docentes uruguayos puntuaron seis puntos más que los españoles.

No debemos obviar en estos temas la consideración de la sociedad en que se hallan insertos los participantes, la historia de algunos temas relativos a la diversidad familiar y su reconocimiento a nivel legislativo. Las respuestas que indican una mayor aceptación del divorcio por parte de los docentes 
uruguayos respecto a los del estudio español pueden estar vinculadas a la larga historia de divorcio en nuestro país, cuya primera ley data de 1907. En ese sentido, Triana y Rodrigo (2010) nos advierten sobre el efecto de la cultura, que consiste en la interpretación y actitudes particulares hacia determinadas configuraciones familiares, a la existencia de mitos sobre su dinámica familiar y a las posibilidades de adaptación de sus miembros en un momento histórico dado. Tómense en cuenta el estudio pionero de Santrock y Tracy (1978), ya mencionado, en el que los participantes partían de una hipótesis de déficit, señalando en sus historias que un hijo de familia que había transitado un divorcio estaba en desventaja respecto de los hijos de familias nucleares.

Visto el aumento de diversidad familiar en Uruguay, del que informáramos, y teniendo en cuenta la universalidad y obligatoriedad de la educación primaria, la escuela se vuelve un ámbito particularmente interesante para trabajar la inclusión de las diferentes configuraciones.

Consideramos que esto se puede lograr con la readecuación de la currícula y la incorporación de temáticas que permitan revisar las ideas que tienen los docentes sobre las familias desde su formación. También sería necesario trabajar en la adaptación de los centros educativos a partir de la diversidad, incorporando material educativo, revisando las comunicaciones que se realizan a las familias, modificando en el calendario algunas celebraciones, entre otros cambios, permitiendo de esta manera que todos los estudiantes se sientan considerados. Parece importante entonces incorporar temáticas sobre diversidad familiar en forma transversal, dialogando con los alumnos, siempre atendiendo al nivel de clase y respetando su derecho a la privacidad (Pichardo, 2011). Todas estas iniciativas necesitan de profesionales formados, que hayan tenido oportunidad de entablar contacto con últimas investigaciones sobre la temática y, también, que hayan podido repasar sus propias historias y vivencias familiares (Deslandes, 2005; Petricone, 2009, Pichardo, 2011). 
La formación continua aparece como una vía para actualizar y fortalecer a los docentes en su contacto diario con las familias, brindándoles herramientas específicas para la atención de la diversidad familiar que hoy puebla las aulas de las escuelas (González Tornaría et. al., 2015). En este sentido, Madureira y Branco (2015) refieren la importancia de llevar a cabo la inclusión y capacitación en el pregrado docente de temas tan sensibles como género, sexualidad y diversidad, ya que se ha visto que, ante la falta de formación, los docentes se manejan con sus opiniones personales y sus experiencias.

Debido al carácter exploratorio del estudio, los datos que presentamos deben ser leídos con cautela. Nos permiten acercarnos a la temática, y podrían servir como un primer paso para futuras investigaciones en las que se pueda trabajar con muestras representativas de la población docente e incorporar otros diseños y técnicas. En este sentido, supone un interesante aporte en el conocimiento de las ideas de docentes sobre la diversidad familiar, alertándonos sobre la necesidad de profundizar en su estudio con el objetivo de señalar la importancia del tema e instrumentar acciones en nuestro país.

\section{Referencias}

Administración Nacional de Educación Pública. Consejo de Formación en Educación. (2010). Modificación del Plan 2008 de Magisterio (Generación 2010). Recuperado de http://www.cfe. edu.uy/images/stories/pdfs/planes_programas/magisterio/2008/ plan2008_modificado_2010.pdf

Administración Nacional de Educación Pública. Consejo Directivo Central. Programa de Lectura y Escritura en Español (2012a). Biblioteca mínima para Educación Inicial y Primaria. Recuperado de http:// www.uruguayeduca.edu.uy/repositorio/prolee/pdf/biblioteca_minima_ educacion_inicial_primaria.pdf

Administración Nacional de Educación Pública. Consejo Directivo Central. Programa de Lectura y Escritura en Español (2012b). Escuela y Familia. Inclusión en la Lectura Letrada. Recuperado de http:// 
www.uruguayeduca.edu.uy/repositorio/prolee/pdf/escuela_y_familiainclusion_en_la_cultura_letrada.pdf

Administración Nacional de Educación Pública. Consejo de Formación en Educación, (2015). Recuperado de http://www.anep.edu.uy/anep/ index.php/noticias/1873-mas-del-60-de-los-alumnos-de-magisterio-yprofesorado-alterna-el-estudio-con-el-trabajo

Antolín, L., Oliva, A., \& Arranz, E. (2009). Variables familiares asociadas a la conducta antisocial infantil: el papel desempeñado por el tipo de estructura familiar. Apuntes de Psicología, 27(2), 1-13.

Arranz, E., \& Oliva, A. (2010). La influencia del contexto familiar en el desarrollo psicológico durante la infancia y la adolescencia. En: E. Arranz Freijo y Oliva A. Delgado (Coords.), Desarrollo psicológico en las nuevas estructuras familiares (pp. 15-27). Madrid: Pirámide.

Arranz, E., Oliva, A., Olabarrieta, F., \& Antolín, L. (2010). Análisis comparativo de las nuevas estructuras familiares como contextos potenciadores del desarrollo psicológico infantil. Infancia y Aprendizaje, 33(4), 503-513. http://dx.doi.org/10.1174/021037010793139653

Arranz, E., Oliva, A., Martin, J. L., \& Parra, A. (2010). Análisis de los problemas y necesidades educativas de las nuevas estructuras familiares. Intervención Psicosocial, 19(3), 243-251. http://dx.doi.org/10.5093/ in2010v19n3a5

Bernedo, I., Fuentes, M. J., Fernández-Molina, M., \& Bersabé, R. (2007). Percepción de las estrategias de socialización parentales en familias adoptivas y no adoptivas. Psicothema, 19(4), 597-602.

Bucheli, M., \& Vigorito, A. (2015). Después de la ruptura: efectos de la separación en los contactos entre padres e hijos y en el bienestar de las mujeres. En M. Bucheli, W. Cabella, M. Nathan, P. Fitermann, A. Vigorito y M Zerpa (Eds.), Cambio familiar y bienestar de las mujeres y los niños en Montevideo y Área Metropolitana. Una perspectiva longitudinal (pp. 43-63). Montevideo: Universidad de la República y UNICEF.

Cabella, W. (2007). El cambio familiar en Uruguay: una breve reseña de las tendencias recientes. Montevideo: UNFPA.

Cabella, W., Fernández Soto, M., \& Prieto, V. (2015). Atlas sociodemográfico $y$ de la desigualdad en el Uruguay. Fascículo 6. Las transformaciones 
de los hogares uruguayos vistos a través de los censos de 1996 y 2011. Montevideo: Ediciones Trilce.

Ceballos-Fernández, M. (2009). La educación formal de los hijos e hijas de familias homoparentales: familia y escuela a contracorriente. Aula Abierta, 37(1), 67-78.

Ceballos-Fernández, M. (2012). Ser madres y padres en familias homoparentales: Análisis del discurso de sus percepciones sobre la educación de sus hijos e hijas. ENSAYOS, Revista de la Facultad de Educación de Albacete, 27, 143-158.

Comellas, M. J. (2009). Familias y escuelas: compartir la educación. Barcelona: Grao.

Day, C. (2005). Formar docentes. Cómo, cuándo y en qué condiciones aprende el profesorado. Madrid: Narcea.

Deslandes, R. (2005). Ecoles, familles, communautés, et pluriethnicité. Plan de Cours, Pédagogie, Premier Cycle, Université de Québec à Trois Rivières.

Golombok, S. (2006). Modelos de familia. ¿Qué es lo que de verdad cuenta? España: Grao.

González, M. M., Chacon, F., Gómez, A. B., Sánchez, M. A, \& Morcillo, E. (2002). Dinámicas familiares, organización de la vida cotidiana y desarrollo infantil y adolescente en familias homoparentales. En: Estudios e investigaciones 2002 (pp. 521-606). Madrid: Oficina del Defensor del Menor de la Comunidad de Madrid.

González, M. M., \& López, F. (2009). Relaciones familiares y vida cotidiana de niños y niñas que viven con madres lesbianas o padres gay. Cultura y Educación, 21(4), 417-428. http://dx.doi. org/10.1174/113564009790002364

González Tornaría, M. L., Wagner, A., \& Saraiva Junges, L. (2015). La colaboración entre familias y escuelas. Una guía para docentes. Montevideo: Magro.

Instituto Nacional de Estadística (1996). VII Censo General de Población y $V$ de Viviendas. Recuperado de: http://www.3.ine.gub.uy/anda4/ index.php 
Instituto Nacional de Estadística (2011): Resultados del Censo de Población 2011: población, crecimiento y estructura por sexo y edad. Recuperado de: http://www.ine.gub.uy/web/guest/censos

Jociles, M. I., Rivas, A. M., Moncó, B., Villamil, F., \& Díaz, P. (2008). Una reflexión crítica sobre la monoparentalidad: El caso de las madres solteras por elección. Portularia, 8(1), 265-274.

López, F., Díez, M., Morgado, B., \& González, M.M. (2008). Educación infantil y diversidad familiar. XXI Revista de Educación, 10, 111-122.

Madureira, A. F. A. y Branco, A. U. (2015). Género, Sexualidade e Diversidade na Escola a partir da Perspectiva de Professores/as. Temas em Psicologia, 23(3), 577-591. http://dx.doi.org/10.9788/TP2015.3-05

Marcondes, K. H. B., \& Sigolo, S. R. R. L. (2012). Comunicação e Envolvimento: Possibilidades de Interconexões entre Famíliaescola? Paidéia, 22(51), 91-99. http://dx.doi.org/10.1590/S0103863X2012000100011

Mello, L., Grossi, M., \& Uziel, A. P. (2009).AEscola e@s Filh@s de Lésbicas e Gays: reflexões sobre conjugalidade e parentalidade no Brasil. En R. Diniz, (Ed.), Diversidade Sexual na Educação: problematizações sobre a homofobia nas escolas (pp. 158-181) Brasilia: Ministério da Educação, Secretaria de Educação Continuada, Alfabetização e Diversidade, UNESCO.

Moliner, L., \& Francisco, A. (2016). 2 mamás y un hijo biológico: trabajando la diversidad familiar en la formación de maestros/as. Revista Nacional e Internacional de Educación Inclusiva, 9(2), 137-154.

Morgado, B., Jiménez-Lagares, I., \& González, M. M. (2009). Ideas del profesorado de primaria acerca de la diversidad familiar. Cultura y Educación, 21(4), 441-451. http://dx.doi. org/10.1174/113564009790002373

Nathan, M., \& Zerpa, M. (2015). Las mujeres y sus hogares. En: M. Bucheli, W. Cabella, M. Nathan, P. Fitermann, A. Vigorito, y M. Zerpa (Eds.), Cambio familiar y bienestar de las mujeres y los niños en Montevideo y Área Metropolitana. Una perspectiva longitudinal (pp. 15-40). Montevideo: Universidad de la República, UNICEF. 
Núñez, L. (2003). Relación familia-escuela. Fracaso eEscolar. En: E. Gervilla (Coord.). Educación familiar. Nuevas relaciones humanas y humanizadoras (pp. 121-130). Madrid: Narcea.

Oliva, A., \& Arranz, E. (Coord.) (2011). Nuevas Familias y Bienestar Infantil. España: Secretariado de Publicaciones de la Universidad de Sevilla, Servicio Editorial de la Universidad del País Vasco.

Oliva, A., \& Palacios, J. (2005). Familia y escuela: padres y profesores. En: M. J. Rodrigo, y J. Palacios (Coords.). Familia y desarrollo humano (pp. 333-350). Madrid: Alianza.

Perrenoud, Ph. (2004). Diez nuevas competencias para enseñar. Madrid: Grao.

Petricone, F. (2009). La familia de origen del docente: Estilo educativo y aspectos relacionados con su ejercicio profesional. En: J. A. Ríos (Ed.), Personalidad, Madurez Humana y Contexto Familiar (pp. 1074-1085). Madrid: Editorial CCS.

Pichardo, J. I. (2011). Diversidad familiar, homoparentalidad y educación. Cuadernos de Pedagogía, 414, 41-44.

Rodrigo, M. J. (2001). Pautas familiares de riesgo y su influencia sobre el desarrollo infantil. BSCP Can Ped, 26(1), 1-4.

Rodrigo, M. J., \& Acuña, M. (2005). El escenario y el currículum educativo familiar. En: M. J. Rodrigo y J. Palacios. Familia y desarrollo humano (pp. 261-276). Madrid: Alianza.

Rosenthal, R., \& Jacobson, L. (1968). Pygmalion in the classroom. Nueva York: Holt, Rinehart \& Winston.

Santrock, J. V., \& Tracy, R. L. (1978). Effects of children's family structure status on the development of stereotypes by teachers. Journal of Educational Psychology, 70, 754-757. http://dx.doi.org/10.1037/00220663.70.5.754

Senado y Cámara de Representantes de la República Oriental del Uruguay (2008). Ley 18246, Unión Concubinaria. Recuperado de http://tbinternet. ohchr.org/Treaties/CCPR/Shared\%20Documents/URY/INT_CCPR_ ADR_URY_14913_S.pdf 
Senado y Cámara de Representantes de la República Oriental del Uruguay (2013). Ley 19075, Matrimonio Igualitario. Recuperado de http:// archivo.presidencia.gub.uy/sci/leyes/2013/05/mec_913.pdf

Torío, S. (2004). Familia, Escuela y Sociedad. Aula Abierta, 83, 35-52.

Triana, B., \& Rodrigo, M. J. (2010). Modelos y estrategias de intervención ante la diversidad familiar. En: E. Arranz Freijo y A. Oliva Delgado (Coords.). Desarrollo psicológico en las nuevas estructuras familiares (pp. 121-142). Madrid: Pirámide. 